\title{
Transport fingerprints at graphene superlattice Dirac points induced by a boron nitride substrate
}

\author{
Rafael Martinez-Gordillo, ${ }^{1,2}$ Stephan Roche, ${ }^{1,3}$ Frank Ortmann,,${ }^{1,4,5}$ and Miguel Pruneda ${ }^{1,2}$ \\ ${ }^{1}$ ICN2_Institut Catala de Nanociencia i Nanotecnologia, Campus UAB, 08193 Bellaterra (Barcelona), Spain \\ ${ }^{2}$ CSIC - Consejo Superior de Investigaciones Cientificas, ICN2 Building, Campus UAB, 08193 Bellaterra (Barcelona), Spain \\ ${ }^{3}$ ICREA-Institucio Catalana de Recerca i Estudis Avancats, 08010 Barcelona, Spain \\ ${ }^{4}$ Institute for Materials Science and Max Bergmann Center of Biomaterials, TU Dresden, 01062 Dresden, Germany \\ ${ }^{5}$ Dresden Center for Computational Materials Science (DCCMS), TU Dresden, 01062 Dresden, Germany \\ (Received 17 September 2013; revised manuscript received 14 March 2014; published 2 April 2014)
}

\begin{abstract}
We report peculiar transport fingerprints at the secondary Dirac points created by the interaction between graphene and boron nitride layers. By performing ab initio calculations, the electronic characteristics of the moiré patterns produced by the interaction between layers are first shown to be in good agreement with experimental data, and further used to calibrate the tight-binding model implemented for the transport study. By means of a real-space order- $N$ quantum transport (Kubo) methodology, low-energy (Dirac point) transport properties are contrasted with those of high-energy (secondary) Dirac points, including both Anderson disorder and Gaussian impurities to respectively mimic short-range and long-range scattering potentials. Mean free paths at the secondary Dirac points are found to range from $10 \mathrm{~nm}$ to a few hundreds of nm depending on the static disorder, while the observation of satellite resistivity peaks depends on the strength of quantum interferences and localization effects.
\end{abstract}

DOI: 10.1103/PhysRevB.89.161401

PACS number(s): 81.05.ue, 72.80.Vp, 73.22.Pr

The understanding of the low-energy transport physics in graphene has recently been the subject of intense debate. In the presence of electron-hole puddles (produced by screened trapped charges), the electronic conductivity exhibits a minimum at the Dirac point (almost temperature independent) which contradicts the scaling theory of localization in twodimensional systems [1], and has been analyzed in terms of percolation theory $[2,3]$.

Perfectly flat hexagonal boron nitride $(h-\mathrm{BN})$ has proved to be an excellent substrate for graphene devices [4] enabling very high mobilities, comparable to those of suspended graphene, thanks to a strong reduction of electron-hole charge fluctuations. However, this also results in a large enhancement of the low-energy resistivity, with a puzzling power-law temperature dependence, hardly described by the conventional Anderson localization regime [5,6]. Different scenarios have been proposed to explain this anomalous feature, such as a semiclassical behavior of the resistivity in ultraclean samples with vanishing density of states, or an unconventional localization regime driven by defect-induced zero-energy modes [7-9].

Although $h$-BN couples only weakly to graphene, the $1.8 \%$ lattice mismatch between them, and any rotational orientation of their lattices give rise to moiré patterns that are observed by scanning tunneling microscopy $[10,11]$. The presence of these patterns suggests that $h$-BN generates a superimposed periodic potential on graphene, significantly modifying its electronic spectrum, as evidenced by the formation of two electron-hole symmetric high-energy secondary Dirac points [12], revealed in both atomic-scale scanning tunneling spectroscopic measurements [11], as well as in various mesoscopic transport measurements focusing on the formation of a fractal energy spectrum (Hofstadter's butterfly) in the presence of strong external magnetic fields [13-16]. The zero-field resistivity fingerprints of those secondary Dirac points remain, however, elusive, varying significantly from sample to sample, with values ranging from a few $\mathrm{k} \Omega$ to about $15 \mathrm{k} \Omega$. The ratio between primary and secondary Dirac point resistivity is also not understood, is sample dependent, and lacks an understanding regarding the role of localization effects in the absence of more in-depth temperature-dependent studies.

This Rapid Communication describes some fundamental transport features at zero-energy and high-energy Dirac points induced by the moiré superlattice potential, which are revealed by varying the nature (short versus long range) and the strength of a superimposed (static) disorder potential. The electronic coupling between graphene and $h$-BN layers is first investigated with $a b$ initio calculations, providing a more realistic and complete description of the generated moiré potential, which can further be contrasted with simplified tightbinding (TB) models. The transport methodology is based on the Kubo-Greenwood formalism, successfully implemented and validated for other forms of disordered graphene [17,18]. Additional weak scatterers are introduced using either the Anderson disorder or the Gaussian potential, as generic shortrange and long-range disorders, respectively [19-25]. The mean free paths obtained are compared with the resistivity behaviors, pinpointing some general reinforcement of the resistance in the vicinity of all Dirac points, whose values and observability are, however, affected by the disorder nature and the strength of quantum interferences. These results provide some guidance for further exploration of high-energy Dirac point transport under a controlled environment.

Electronic properties of graphene on boron nitride substrates. Previous theoretical calculations based on density functional theory (DFT) assumed perfectly eclipsed honeycomb lattices of both two-dimensional (2D) structures, neglecting any mismatch in their lattice constants. This approximation breaks the symmetry of the two sublattices in graphene, opening a band gap on the order of $50 \mathrm{meV}$, which disagrees with most experimental data (although Hunt et al. [15] observe gap formation in their transport measurements for samples with long wavelengths, and suggest that manybody interactions may play a role; see the discussion in the Supplemental Material [26]). Here, we explicitly consider the somewhat larger lattice constants of $h$-BN compared to graphene and performed DFT calculations for three different 
(a)

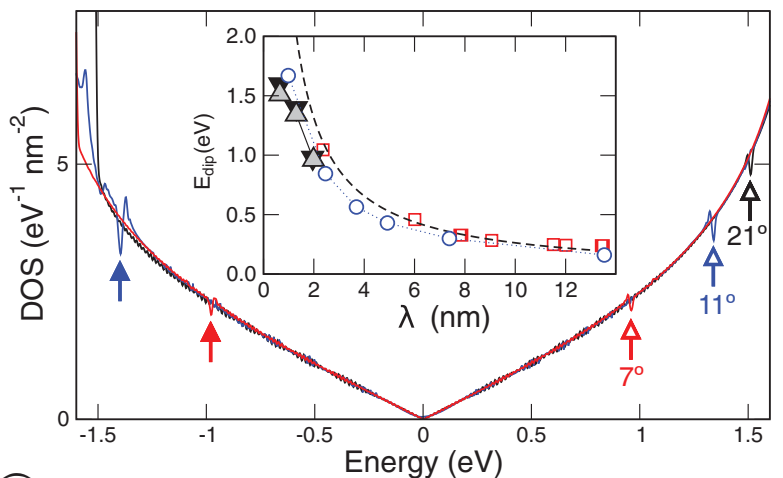

(b)

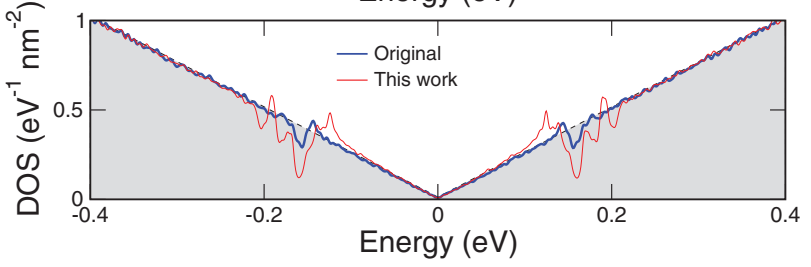

(c)

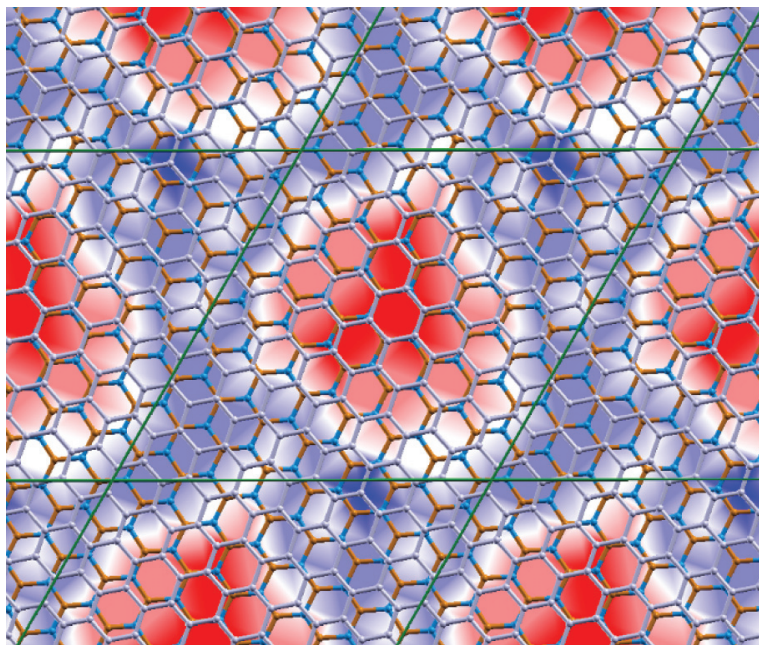

FIG. 1. (Color online) (a) Main frame: Density of states computed from DFT for moiré lattices with angles of $7^{\circ}, 11^{\circ}$, and $21^{\circ}$. The arrows indicate the positions of the dips getting closer to zero energy as the angle is decreased. Inset: Dependence of dip energy on the wavelength $\lambda$. Black down (up) triangles are first-principles data with the dip below (above) the Fermi level. Blue circles are data from the diagonalized TB model. The dashed line is the expected theoretical value ( $E_{\text {dip }}$; see text). Red squares are experimental data from Ref. [11]. (b) DOS from the TB model including the moiré potential using the original parameters of Ref. [29] (thick blue line) compared to threefold magnified potential (thin red line), and the DOS of pristine graphene (dashed black line). (c) Energy profile representing the sublattice antisymmetric part of the potential $\left(m^{*}\right)$ for the moiré superlattice with $\phi=7^{\circ}$.

moiré patterns, corresponding to rotation angles of $7^{\circ}, 11^{\circ}$, and $21^{\circ}$ between graphene and $h$-BN lattices. The wavelengths of these patterns $\lambda(1.95,1.30$, and $0.65 \mathrm{~nm})$ require supercells with 248, 110, and 28 atoms, respectively. The DFT calculations, which explicitly include van der Waals interactions, were performed using the SIESTA code $[27,28]$.

Figure 1(a) shows the density of states (DOS) for the three moiré structures with emerging superlattice features (dips) indicated by arrows. These dips, which appear almost symmetrically above and below the charge neutrality point,
TABLE I. Moiré potential parameters extracted from DFT result.

\begin{tabular}{lccccc}
\hline \hline Angle $(\mathrm{deg})$ & $A(\mathrm{meV})$ & $B(\mathrm{meV})$ & $C(\mathrm{meV})$ & $\phi_{1}$ & $\phi_{2}$ \\
\hline 21 & 19 & 14 & 4 & 5.8 & 3.9 \\
11 & 32 & 23 & 2 & 0.7 & 0.1 \\
7 & 39 & 30 & 0 & 3.5 & 4.0 \\
\hline \hline
\end{tabular}

are due to the new Dirac states induced by the underlying $h$-BN superlattice potential. Their energy depends on the moiré periodicity [inset of Fig. 1(a)], in good agreement with theory $\left(E_{\text {dip }}=2 \pi \hbar v_{F} / \sqrt{3} \lambda\right)$ and experimental literature [11]. Notice that a small electron-hole asymmetry appears due to the interlayer interaction, but there is no band gap opening at the primary Dirac point within our numerical resolution $(8 \mathrm{meV})$.

The electronic properties of graphene on top of the polar substrate can be reasonably described with a simplified tightbinding model

$$
\hat{H}=v \mathbf{p} \cdot \sigma+m^{*}(\mathbf{r}) \sigma_{z}+n(\mathbf{r}) \mathbb{I},
$$

where the first term stems from pristine graphene while the second and third terms take into account the substrate influence. The Pauli matrices $\sigma=\left(\sigma_{x}, \sigma_{y}, \sigma_{z}\right)$ operate on the basis of the two graphene sublattices, $\mathbf{p}$ is the momentum in the plane, $m^{*}(\mathbf{r})$ is an effective-mass term that breaks the sublattice symmetry locally, and $n(\mathbf{r})$ is a long-range potential symmetric in the sublattices. Both $m^{*}$ and $n$, have the periodicity of the moire pattern, $\lambda$. We quantify the strength of the substrate potential from the values of the local energies in our DFT calculations, which can be estimated, in analogy to the Mulliken charges, from $E_{i}=\sum_{\mu^{\prime} v} \rho_{\mu \nu} H_{\nu \mu}$, where $\hat{\rho}$ is the electronic density matrix, $\hat{H}$ is the Hamiltonian matrix, $\mu, v$ denote localized atomic orbitals, and the primed sum runs over all orbitals $\mu$ belonging to atom $i$. We focus only on the changes in the local energy induced by the $h$-BN substrate by taking $\Delta E_{i}=E_{i}^{\mathrm{CBN}}-E_{i}^{\mathrm{C}}$, where $E_{i}^{\mathrm{CBN}}$ and $E_{i}^{\mathrm{C}}$ denote the local energies on $\mathrm{C}$ atoms in the moire $\mathrm{C} / \mathrm{BN}$ pattern and in isolated graphene, respectively. As an illustration, Fig. 1(c) shows the antisymmetric part $\left(m^{*}\right)$ of the local potential $\Delta E_{i}$ for the moiré lattice with wavelength of $1.95 \mathrm{~nm}$.

We next parametrize the effective moiré potential using the simplified expression proposed by Sachs et al. [29]:

$$
m^{*}=\frac{1}{2}\left[A \sin \left(\frac{2 \pi x}{\lambda}+\phi_{1}\right)+B \sin \left(\frac{2 \pi y}{\lambda}+\phi_{2}\right)+C\right] \text {. }
$$

The average value of the modulated mass term $m^{*}(\mathbf{r})$ (given by $C$ ) is related to the width of the band gap of the system (which we find to be negligibly small in our calculations). We also find that the corrugation height of $m^{*}(\mathbf{r})$ (determined by the $A$ and $B$ parameters reported in Table I) depends on the rotation angle (being stronger for larger wavelengths) and on the interlayer distance (stronger for shorter distances), and can be larger than previously reported. ${ }^{1}$

\footnotetext{
${ }^{1}$ The parameters given in Ref. [29] are $A=18.6 \mathrm{meV}, B=$ $42.0 \mathrm{meV}, \phi_{1}=1.884$, and $\phi_{2}=1.531$, and were obtained from DFT calculations considering different stacking configurations of graphene on top of $h$-BN.
} 
Further increase in the strength of the underlying potential can be expected from thicker $h$-BN layers above or below the graphene sheet. Such variability in the values of $A$ and $B$ translates into changes in the depth of the dips in the DOS related to the secondary Dirac points, which is demonstrated in Fig. 1(b). On the other hand, the energy position of these Dirac points is unchanged with respect to the intensity of the potential and follows the expected dependence with $\lambda\left(E_{\text {dip }}\right)$, as shown in the inset of Fig. 1(a). Since secondary Dirac points are more easily accessible by gating when they occur at moderate energies $(\sim 0.2 \mathrm{eV})$, in the following we focus on the transport properties of the graphene/ $h$-BN structure with the largest possible moiré periodicity $(\sim 14 \mathrm{~nm})$, which is out of reach for first-principles simulations. To better contrast the superlattice potential effects with experimental transport data, we employ the tight-binding model with values of $A=56 \mathrm{meV}$ and $B=126 \mathrm{meV}$ [26].

Quantum transport methodology and results. The quantum wave packet dynamics and Kubo conductivity are calculated using a real-space implementation and order- $N$ algorithms (Lanczos approach) $[17,18,25,30]$. The dc conductivity $\sigma(E, t)$ for energy $E$ and time $t$ is given by $\sigma(E, t)=$ $e^{2} \rho(E) \Delta X^{2}(E, t) / t$, with $\rho(E)$ being the DOS and $\Delta X^{2}(E, t)$ the mean quadratic displacement of the wave packet at energy $E$ and time $t$ :

$$
\Delta X^{2}(E, t)=\frac{\operatorname{Tr}\left[\delta(E-\mathcal{H})|\hat{X}(t)-\hat{X}(0)|^{2}\right]}{\operatorname{Tr}[\delta(E-\mathcal{H})]}
$$

A key quantity in the analysis of the transport properties is the diffusion coefficient $D(E, t)=\Delta X^{2}(E, t) / t . D(t)$ exhibits a transition from a short-time ballistic motion to a saturation regime, from which the mean free path $\ell_{e}$ is extracted from $\ell_{e}(E)=D^{\max }(E) / 2 v(E)[v(E)$ is the velocity derived at short times]. The semiclassical conductivity $\sigma_{s c}$ is given by $\frac{1}{2} e^{2} \rho(E) D^{\max }(E)$ and the semiclassical resistivity $R_{s c}=$ $1 / \sigma_{s c}(E)$. The contribution of quantum interferences is further indicated by a time-dependent decay of $D(E, t)$ for long elapsed times. The spin degree of freedom is included as a factor of 2 for $\sigma$ and $\rho$, while calculations are performed with systems of several tens of millions of carbon atoms and energy resolution down to $0.54 \mathrm{meV}$. A modulation of the potential profile is introduced on top of the above described tightbinding model by taking on-site energies at random within $\left[-0.25 \gamma_{0}, 0.25 \gamma_{0}\right]$, a common model for short-range scattering potentials $[1,19]$, with $\gamma_{0}=-2.7 \mathrm{eV}$ the nearest-neighbor hopping. The Gaussian disorder is defined by a chosen density of Coulomb impurities $(0.125 \%)$ and a long-range scattering potential, following commonly used parameters to mimic screened charges trapped in the substrate [18,20-23]. The contribution from $N_{I}$ impurities randomly distributed at $\mathbf{r}_{i}$ is given by renormalized on-site energies at orbital $\alpha$ with $\varepsilon_{\alpha}=\sum_{i=1}^{N_{I}} \varepsilon_{i} \exp \left[-\left|\mathbf{r}_{\alpha}-\mathbf{r}_{i}\right|^{2} /\left(2 \xi^{2}\right)\right]$, where $\xi=0.426 \mathrm{~nm}$, defines the effective potential range, while $\varepsilon_{i}$ are chosen at random within $\left[-W \gamma_{0} / 2, W \gamma_{0} / 2\right]$, with $W$ monitoring the total disorder strength (we choose two representative values $W=1$ and $W=2$ for introducing weak and strong intervalley scattering, respectively). The results presented below are obtained by averaging over six different random configurations, for both Anderson disorder and Gaussian

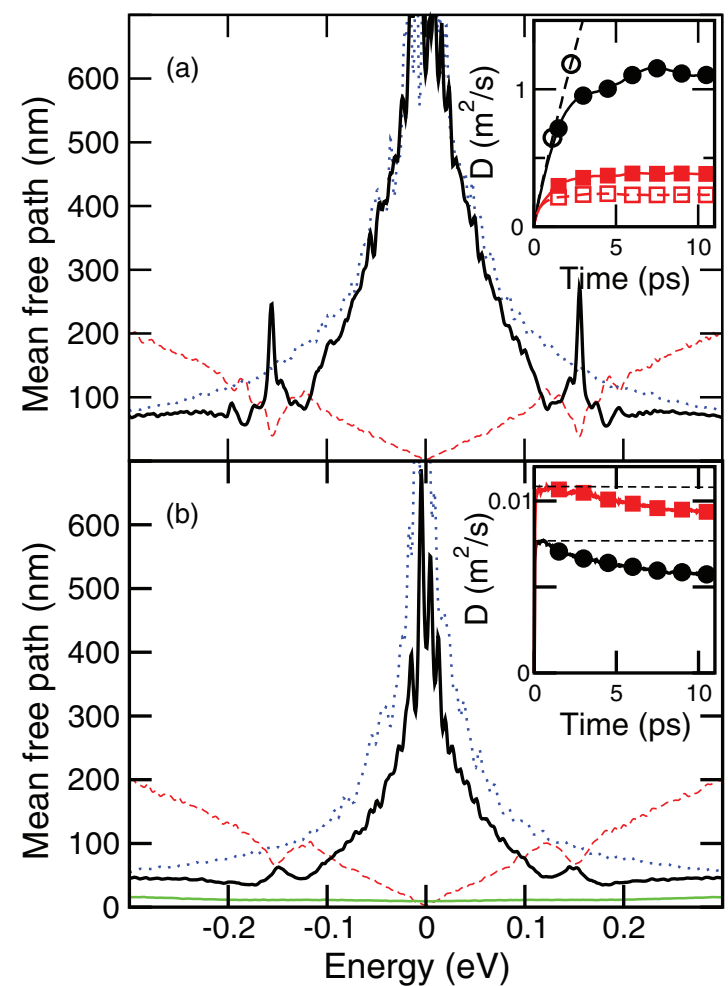

FIG. 2. (Color online) Mean free path for disordered graphene without (dotted blue) and including the moiré potential $m^{*}(\mathbf{r})$. (a) Case with Anderson disorder for $W=0.5$ (solid black), and (b) Gaussian long-range disorder for $W=1$ (solid black) and $W=2$ (solid green). Rescaled DOSs are shown as dashed red lines. Inset (a) $D(E=0, t)$ (black circles) and $D\left(E=E_{\mathrm{dip}}, t\right)$ (red squares) for Anderson disorder with moiré potential (filled) and without moiré potential (empty). Inset (b) $D(E=0, t)$ (black circles) and $D\left(E=E_{\text {dip }}, t\right)$ (red squares) for the case $W=2$. The dashed lines correspond to the maximum diffusion for each case.

impurities. The energy scale of the disorder for the Anderson model $(\sim 0.67 \mathrm{eV})$ and the Gaussian model $(2.7 \mathrm{eV})$ is much bigger than the modulation of $n(\mathbf{r})\left(\sim 6 \mathrm{meV}\right.$, for $\left.7^{\circ}\right)$, which can therefore be neglected as an additional long-range and sublattice-symmetric contribution.

Figure 2 shows the mean free paths computed for unsupported graphene, compared to those of graphene on top of $h$-BN with superimposed short-range Anderson (a) and long-range Gaussian (b) disorder potentials. Mean free paths are generally seen to strongly increase close to the zero-energy Dirac point, as well as in the vicinity of the secondary Dirac point (in the presence of the moiré superlattice potential), although the amplitudes depend on the nature (short versus long range) and intensity of the scattering potential. For longrange disorder, $\ell_{e}(E)$ is found to be always larger inthe absence of the moiré potential, whereas the case of short-range disorder exhibits some opposite trend in the vicinity of secondary Dirac points. Notice that for most of the chosen disorder parameters, localization effects remain extremely weak [as evidenced by a saturation behavior of the diffusion coefficient, Fig. 2(a) inset], except for a strong on-site impurity potential [illustrated in the case of Gaussian impurities and $W=2$, Fig. 2(b) inset], for which an energy-independent $\ell_{e}(E) \sim 10 \mathrm{~nm}$ is obtained 


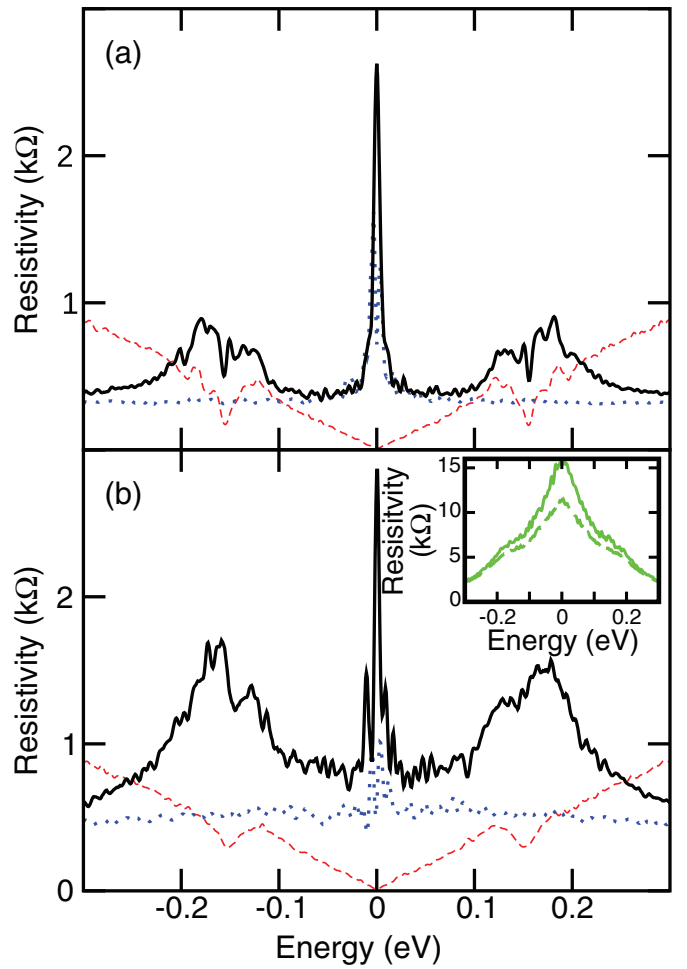

FIG. 3. (Color online) Resistivity with (solid black) and without (dotted blue) moiré potential. (a) Case with Anderson disorder ( $W=$ 0.5 ). (b) Case with Gaussian potential $(W=1)$. The rescaled DOS is shown for comparison (dashed red). Inset (b) Resistivity for the case $W=2$ using the semiclassical expression (dashed) and the quantum resistivity at time $t=10 \mathrm{ps}$ (solid).

[green solid line in Fig. 2(b)]. For such disorder strength, as seen in Fig. 2(b) (inset), the diffusion coefficient shows strong decay after reaching its maximum value, pinpointing the emergence of quantum interferences.

The corresponding behaviours of the semiclassical resistivity are reported in Fig. 3. The enhancement of the mean free path in the vicinity of the high-energy Dirac points is seen to be transformed into a reinforcement of the resistivity, which stems from the strongly reduced density of states. In addition to the standard zero-energy peak of the longitudinal resistivity, two satellite resistivity peaks are formed in the presence of the substrate-induced moiré potential, in clear agreement with experimental results [13-15]. For low enough disorder, the transport regime at the secondary Dirac points as well as at the primary Dirac point remain, however, far from a strong insulating regime given the large values of $\ell_{e}(E) \in$ [80 nm, $1 \mu \mathrm{m}$ ], suggesting localization lengths several microns long [17], and therefore limiting the observation of variablerange hopping to vanishingly small temperatures [31].

The satellite resistivity peaks therefore remain observable as long as quantum interferences are negligible, whereas the emergence of localization effects is concomitant with the vanishing of transport signatures of the secondary Dirac points. This is illustrated in Fig. 3(b) when varying the disorder strength from $W=1$ to $W=2$. For $W=2$, as seen in Fig. 2(b) (inset), the diffusion coefficient exhibits a marked time-dependent decay after initial saturation, evidencing the significant contribution of quantum interferences induced by intervalley scattering [24]. The corresponding resistivities reported in Fig. 3(b) (inset) show no traces of secondary Dirac point, but an increasing resistivity with enhancement of coherent localization effects (the dashed line gives $R_{s c}$ whereas the solid line denotes the quantum resistivity computed at a length scale longer than $\ell_{e}$, that is, including the localization contribution).

In conclusion, fundamental transport features in graphene induced by a moiré superlattice potential have been unraveled. At the energy of the superlattice (secondary) Dirac points, an increase of both the mean free paths and resistivity has been obtained, confirming experimental data but also quantifying the role of superimposed disorder in tuning the relative resistivity between primary and secondary Dirac points. The long localization lengths deduced from the mean free path indicate a modest contribution of quantum interferences and weak intervalley scattering. In contrast, whenever static disorder leads to mean free paths in the order of $10 \mathrm{~nm}$, sizable quantum interferences develop and jeopardize the identification of satellite resistivity peaks. It should be noted that electron-hole asymmetric resistivity peaks, which are usually observed in experiments, could be captured by further adjusting our TB model.

This work was funded by Spanish FEDER-MINECO (Grants No. FIS2009-12721-C04-01 and No. CSD200700041) and AGAUR (Grant No. FI-DGR 2011FI B 00993). We would like to thank the Spanish Supercomputer Network for computing resources. S.R. acknowledges support from the Spanish Ministry of Economy and Competitiveness (under Contract No. MAT2012-33911). The research leading to these results has received funding from the European Union Seventh Framework Programme under Grant Agreement No. 604391 "Graphene Flagship".
[1] F. Evers and A. D. Mirlin, Rev. Mod. Phys. 80, 1355 (2008).

[2] S. D. Sarma, S. Adam, E. Hwang, and E. Rossi, Rev. Mod. Phys. 83, 407 (2011).

[3] V. V. Cheianov, V. I. Falko, B. L. Altshuler, and I. L. Aleiner, Phys. Rev. Lett. 99, 176801 (2007).

[4] C. Dean, A. Young, I. Meric, C. Lee, L. Wang, S. Sorgenfrei, K. Watanabe, T. Taniguchi, P. Kim, K. Shepard et al., Nat. Nanotechnol. 5, 722 (2010).
[5] L. A. Ponomarenko, A. K. Geim, A. A. Zhukov, R. Jalil, S. V. Morozov, K. S. Novoselov, I. V. Grigorieva, E. H. Hill, V. V. Cheianov, V. I. Falko et al., Nat. Phys. 7, 958 (2011).

[6] F. Amet, J. R. Williams, K. Watanabe, T. Taniguchi, and D. Goldhaber-Gordon, Phys. Rev. Lett. 110, 216601 (2013).

[7] V. M. Pereira, F. Guinea, J. M. B. Lopes dos Santos, N. M. R. Peres, and A. H. Castro Neto, Phys. Rev. Lett. 96, 036801 (2006). 
[8] M. M. Ugeda, I. Brihuega, F. Guinea, and J. M. GómezRodríguez, Phys. Rev. Lett. 104, 096804 (2010).

[9] A. Cresti, F. Ortmann, T. Louvet, V. Dinh, and S. Roche, Phys. Rev. Lett. 110, 196601 (2013).

[10] J. Xue, J. Sanchez-Yamagishi, D. Bulmash, P. Jacquod, A. Deshpande, K. Watanabe, T. Taniguchi, P. Jarillo-Herrero, and B. J. LeRoy, Nat. Mater. 10, 282 (2011).

[11] M. Yankowitz, J. Xue, D. Cormode, J. Sanchez-Yamagishi, K. Watanabe, T. Taniguchi, P. Jarillo-Herrero, P. Jacquod, and B. LeRoy, Nat. Phys. 8, 382 (2012).

[12] C.-H. Park, L. Yang, Y.-W. Son, M. L. Cohen, and S. G. Louie, Phys. Rev. Lett. 101, 126804 (2008).

[13] L. A. Ponomarenko, R. V. Gorbachev, G. L. Yu, D. C. Elias, R. Jalil, A. A. Patel, A. Mishchenko, A. S. Mayorov, C. R. Woods, J. R. Wallbank et al., Nature (London) 497, 594 (2013).

[14] C. R. Dean, L. Wang, P. Maher, C. Forsythe, F. Ghahari, Y. Gao, J. Katoch, M. Ishigami, P. Moon, M. Koshino et al., Nature (London) 497, 598 (2013).

[15] B. Hunt, J. D. Sanchez-Yamagishi, A. F. Young, M. Yankowitz, B. J. LeRoy, K. Watanabe, T. Taniguchi, P. Moon, M. Koshino, P. Jarillo-Herrero et al., Science 340, 1427 (2013).

[16] W. Yang, G. Chen, Z. Shi, C.-C. Liu, L. Zhang, G. Xie, M. Cheng, D. Wang, R. Yang, D. Shi et al., Nat. Mater. 12, 792 (2013).

[17] A. Lherbier, B. Biel, Y.-M. Niquet, and S. Roche, Phys. Rev. Lett. 100, 036803 (2008).

[18] S. Roche, N. Leconte, F. Ortmann, A. Lherbier, D. Soriano, and J.-C. Charlier, Solid State Commun. 152, 1404 (2012).

[19] P. W. Anderson, Phys. Rev. 124, 41 (1961).
[20] K. Nomura and A. H. MacDonald, Phys. Rev. Lett. 98, 076602 (2007).

[21] A. Rycerz, J. Tworzydlo, and C. W. J. Beenakker, Europhys. Lett. 79, 57003 (2007).

[22] K. Wakabayashi, Y. Takane, and M. Sigrist, Phys. Rev. Lett. 99, 036601 (2007)

[23] Y.-Y. Zhang, J. Hu, B. A. Bernevig, X. R. Wang, X. C. Xie, and W. M. Liu, Phys. Rev. Lett. 102, 106401 (2009).

[24] F. Ortmann, A. Cresti, G. Montambaux, and S. Roche, Europhys. Lett. 94, 47006 (2011).

[25] T. M. Radchenko, A. A. Shylau, and I. Zozoulenko, Phys. Rev. B 86, 035418 (2012).

[26] See Supplemental Material at http://link.aps.org/supplemental/ 10.1103/PhysRevB.89.161401 for a discussion on the debated gap opening in graphene/ $h$-BN moirés, details of calculations using different potential strengths, and related information.

[27] J. M. Soler, E. Artacho, J. D. Gale, A. García, J. Junquera, P. Ordejón, and D. Sánchez-Portal, J. Phys.: Condens. Matter 14, 2745 (2002).

[28] G. Román-Pérez and J. M. Soler, Phys. Rev. Lett. 103, 096102 (2009).

[29] B. Sachs, T. O. Wehling, M. I. Katsnelson, and A. I. Lichtenstein, Phys. Rev. B 84, 195414 (2011).

[30] L. E. F. Foa Torres, S. Roche, and J. C. Charlier, Introduction to Graphene-Based Nanomaterials: From Electronic Structure to Quantum Transport (Cambridge University Press, Cambridge, UK, 2014).

[31] J. Moser, H. Tao, S. Roche, F. Alzina, C. M. Sotomayor Torres, and A. Bachtold, Phys. Rev. B 81, 205445 (2010). 